\title{
Successful treatment of Cronkhite-Canada syndrome using anti-tumor necrosis factor antibody therapy
}
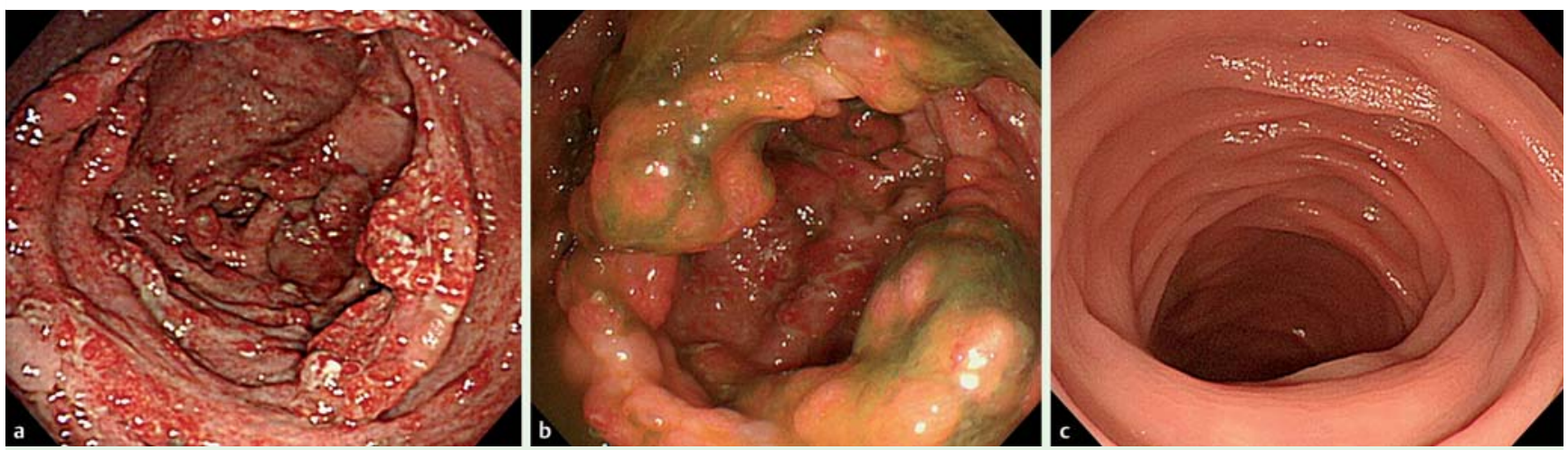

Fig. 1 Colonoscopy in a patient with Cronkhite-Canada syndrome. a Before initial treatment. b 3 days before ileocecal resection. c 20 months after anti-tumor necrosis factor alpha therapy.

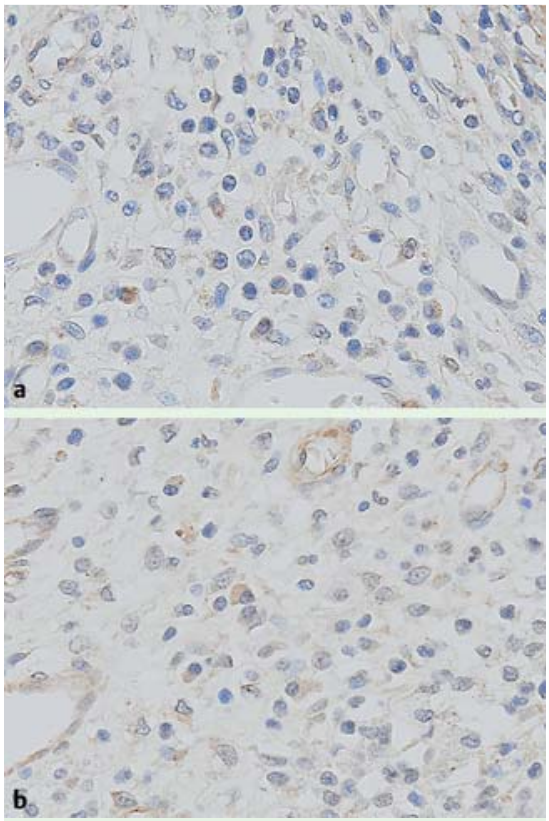

Fig. 2 Immunohistochemistry for tumor necrosis factor alpha (TNF- $\alpha$ ). Colon tissue of patient with Cronkhite-Canada syndrome was stained with: a anti-TNF- $\alpha$ antibody; $\boldsymbol{b}$ isotype control.

Cronkhite-Canada syndrome (CCS) is a rare nonhereditary syndrome characterized by gastrointestinal polyposis and ectodermal changes [1]. Although several treatments, such as steroids, are available, the prognosis is poor, with a 5-year mortality rate of $55 \%$ [2].

A 57-year-old man, who suffered from alopecia, anorexia, severe diarrhea, dystrophic nail changes, and pigmentation on the hands, presented to our hospital.
Colonoscopy revealed numerous polyps throughout the colon ( $\bullet$ Fig. 1 a). Gastroscopy and small-bowel series also showed severe gastric and small-bowel polyposis, and histology showed juvenile-like polyps with mild inflammation. On the basis of these findings, the patient was diagnosed with CCS.

He was treated with medications including corticosteroids, antiplasmin agents, and azathioprine; however, the symptoms persisted. He developed intussusception 7 years after diagnosis ( $\bullet$ Fig. 1 b) and underwent ileocecal resection. As he continued to suffer from frequent relapses, with diarrhea and malnutrition with severe hypoalbuminemia, a different therapeutic strategy was needed to control the disease progression. Supported by a single report finding of high levels of tumor necrosis factor alpha (TNF- $\alpha$ ) in tissue affected by CCS [3], implying that antiTNF- $\alpha$ therapy could be useful for CCS patients, the patient was administered infliximab at $200 \mathrm{mg}$ every 2 weeks. His general condition improved, and colonoscopy performed 20 months after induction of anti-TNF therapy showed complete remission with the disappearance of polyposis ( Fig. 1c). He now receives $200 \mathrm{mg}$ of infliximab every 8 weeks, and has been symptom free for 3 years since the initial administration.

There is one report that described strong TNF- $\alpha$ expression in intestinal mucosa affected by CCS, implicating the potential usefulness of anti-TNF- $\alpha$ antibody for patients with CCS. However, to the best of our knowledge, this treatment has not been tested until now [3]. Although we could not detect TNF- $\alpha$ expression in the polyps of this patient by immunohistochemistry ( $\bullet$ Fig. 2 ), given the patient's dramatic response to therapy, it can be assumed that TNF plays an important role in disease development, perhaps upstream of polyp formation. This case indicates that further basic and clinical research is warranted, and that anti-TNF therapy could be an important new strategy for treatment of CCS.

Endoscopy_UCTN_Code_CCL_1AD_2AC

Competing interests: None

Daisuke Watanabe ${ }^{1}$, Makoto Ooi ${ }^{1}$, Namiko Hoshi ${ }^{1}$, Michitaka Kohashi ${ }^{1}$, Tomoo Yoshie', Nobunao Ikehara', Masaru Yoshida², Emmy Yanagita ${ }^{3}$, Takashi Yamasaki ${ }^{3}$, Tomoo Itoh ${ }^{3}$, Takeshi Azuma ${ }^{1}$

${ }^{1}$ Division of Gastroenterology, Department of Internal Medicine, Kobe University Graduate School of Medicine, Kobe, Japan

2 Division of Metabolomics Research, Department of Internal Medicine, Kobe University Graduate School of Medicine, Kobe, Japan

3 Division of Diagnostic Pathology, Department of Pathology, Kobe University Graduate School of Medicine, Kobe, Japan 


\section{References}

1 Cronkhite LW Jr, Canada WJ. Generalized gastrointestinal polyposis; an unusual syndrome of polyposis, pigmentation, alopecia and onychotrophia. N Engl J Med 1955; 252: $1011-1015$

2 Nakayama M, Muta H, Somada S et al. Cronkhite-Canada syndrome associated with schizophrenia. Intern Med 2007; 46: $175-$ 180

3 Martinek J, Chvatalova T, Zavada F et al. A fulminant course of Cronkhite-Canada syndrome. Endoscopy 2010; 42: E350 - 351
Bibliography

DOI http://dx.doi.org/

10.1055/s-0034-1377539

Endoscopy 2014; 46: E476-E477

(c) Georg Thieme Verlag KG

Stuttgart · New York

ISSN 0013-726X

\section{Corresponding author}

Takeshi Azuma, MD, PhD

Division of Gastroenterology

Department of Internal Medicine

Kobe University

7-5-1, Kusunoki-cho

Chuo-ku, Kobe 650-0017

Japan

Fax: +81-78-3826309

azumat@med.kobe-u.ac.jp 